\title{
Study designs in Paediatric Pharmacoepidemiology
}

Verhamme ${ }^{1}$ KMC, MD, PhD, Sturkenboom ${ }^{1,2}$ MCJM, Pharm D, PhD on behalf of the TEDDY European Network of Excellence.

1 Department of Medical Informatics, Erasmus MC, Rotterdam, The Netherlands

2 Department of Epidemiology, Erasmus MC, Rotterdam, The Netherlands

Corresponding author:

Katia Verhamme

Dr.Molewaterplein 50

3015 GE Rotterdam

Postbus 2040

3000 CA Rotterdam

Tel : $+31 / 107044122$

Fax: $+31 / 107044722$

k.verhamme@erasmusmc.nl

\section{$\underline{\text { Conflict of interest: }}$}

As employees of Erasmus MC, MCJMS and VKMC has been involved as project leader and in analyses contracted by various pharmaceutical companies and received unconditional research grants from Pfizer, Merck, Johnson\&Johnson, Amgen, Roche, GSK, Boehringer, Yamanouchi and Altana. None of which are related to the subject of this paper.

Key words: Pharmacoepidemiology, children, safety, effectiveness, case-control, cohort 


\section{Abstract}

Purpose: Few data on the efficacy and safety of drugs in children are available as in the past, these children were not included in randomized controlled trials. (RCTs) Data on efficacy and safety of drugs in children are extrapolated from adults. The EMEA recognizes the need for long term safety studies on various drugs, a need which can best be answered by pharmacoepidemiological studies. In this article, we provide current available information on study designs within the field of paediatric drug research.

Methods: A PubMed search was conducted on all pharmacoepidemiological studies in children. In addition, data from handbooks on pharmacoepidemiology were consulted. Data were reviewed and relevant literature on study designs in paediatric pharmacoepidemiology is described.

Results: The various study designs in pharmacoepidemiology have their specific indications all with their specific limitations. Case reports and case series are mainly used for signal detection of safety issues whereas case control and cohort studies are used for safety hypothesis testing. Observational studies can be conducted using data from automated databases which guarantee large sample size and long term follow-up which is ideal for safety studies especially in case of rare events.

Conclusion: Pharmacoepidemiological studies are crucial in the research on the safety of drugs in children. Knowledge on the different pharmacoepidemiology methods is important to guarantee optimal use and correct interpretation of the data. 


\section{Introduction}

When doctors prescribe medicines to children, they want these drugs to be effective and safe. So far, few randomized controlled trials (RCTs) have been conducted in children meaning that most of these drugs have only been licensed for use in adults. [1] Data from adults are extrapolated to children although we know that children are "no small adults". Instructions on dosing and route of administration for use in children are derived from the data on adults, although pharmacological and pharmacokinetic studies have shown that children metabolize drugs differently. [2]

Recently, the importance for the need of safe and effective drugs in children has been recognized and this has lead to several initiatives. The new EU paediatric regulation specifies the need for a Paediatric Investigation Plan as a mandatory part for each new licence application and the potential for a Paediatric Use Marketing Authorisation (PUMA) for older off-patent drugs if studies on the efficacy and safety of these older drugs in children will be conducted. [3] Equally, in the US, paediatric research is stimulated via the Pediatric Research Equity Act. [4] In addition, several networks have been established to promote the safety and efficacy of drugs in children. [5]

Demonstration of efficacy is best achieved using RCTs either against placebo or against the current standard of care. The evaluation of safety is often much more complex. Although some of the safety information can be provided by RCTs, they are often limited in terms of sample size and in length of follow-up. If the incidence of an adverse event is very rare or if the adverse event only arises in the long term, this potential safety issue will not be detected through RCTs. In addition, RCTs often use strict in and exclusion criteria meaning that children with specific co-morbidity will be excluded from the trial and that the information of safety and efficacy cannot be generalized to the whole paediatric population. The EMEA list of Paediatric Needs recognizes the need for long term safety studies in children. [6] By definition, these types of studies require a long term follow-up and large sample size to detect rare events. This implicates that paediatric drug safety can best be answered by pharmacoepidemiological studies. Within this article, we provide an overview on the various study designs that can be used in pharmacoepidemiology and where relevant focus on issues related to paediatric research. 


\section{Methods}

A PubMed search was conducted on the following MESH term: "Pharmacoepidemiology" with as limits: English, all infant: birth-23 months, all child: 0-18 years, newborn: birth -1 month, infant: 1-23 months, preschool child: $2-5$ years, child: 6-12 years and adolescent: 13-18 years. This search generated 142 hits (search date May 2009). All abstracts were reviewed and if considered to be relevant - meaning containing information on study designs in pharmacoepidemiology, ideally also touching upon pharmacoepidemiology in children-, the full article was consulted. Apart from PubMed, handbooks on pharmacoepidemiology were as well consulted namely the following: "Textbook of pharmacoepidemiology" by Strom and Kummel and "Pharmacoepidemiology and Therapeutic Risk Management" by Hartzema et al. $[7,8]$ In addition, the authors elaborated on methods that were used to study the safety and efficacy of drugs in children within the scope of the TEDDY (Taskforce in Europe for the Drug Development of the Young) network. [9] The authors in no way intended to conduct a systematic review on methods in pharmacoepidemiology, meaning that certain papers might have been left out based on the decision of the authors. 


\section{What is pharmacoepidemiology?}

Epidemiology is the study of the frequency, distribution and determinants of a disease and goes back to ancient times. [7]

Pharmacoepidemiology is the study of the use and the effects of drugs in large numbers of people. Pharmacoepidemiology implements the basic principles of chronic disease epidemiology in its research methods. In addition, pharmacoepidemiology integrates the pharmacology of a drug in the concept of the different study designs. Pharmacoepidemiology thus bridges between epidemiology and pharmacology. [8]

Awareness of the importance of the safety of a drug and the need of drug regulation is relatively new and goes back to the beginning of the $20^{\text {th }}$ century. Pharmacoepidemiology mainly got his boost in 1961 where growing concerns on the safety of a drug were triggered after the thalidomide disaster. [10] Thalidomide was marketed as a mild hypnotic and often used in pregnant women for the symptomatic relief of morning-sickness. Shortly after marketing, a dramatic increase in the occurrence of phocomelia (absence of limbs or part of limbs) was observerd although phocomelia was known to be a rare birth defect. After pharmacoepidemiological investigation, the increase of phocomelia was found to be associated with in utero exposure to thalidomide.

Pharmacoepidemiology is mainly applied to drugs that have already been registered (postmarketing drug surveillance) but in recent years, the scope of pharmacoepidemiology has broadened considerably. [8]

\section{Importance of pharmacoepidemiology}

As already alluded on in the introduction, it is difficult to study the safety of a drug through RCTs as these trials often use stringent in - and exclusion criteria, have limited sample size and short term follow-up. Especially if adverse events are uncommon, if they only appear after long term follow-up or if they are unique to high risk populations, the chance of detecting them within RCTs is low. Pharmacoepidemiology can contribute information on the safety and effectiveness of a drug that is not available from premarketing studies. [8] A well known example of a pharmacoepidemiological study which was crucial in the detection of a safety issue is the case control study on the association between the use of diethylstilbestrol for the prevention of spontaneous abortion in pregnant women and the occurrence of vaginal cancer in their offspring. [11] As this event only occurred after long term, namely in the female offspring from adolescence on, this signal would never have been picked up by clinical trials. More recent examples of safety signals that were picked up through observational research are the cohort studies on the relation between the use of cyclooxygenase2- selective non steroidal anti-inflammatory drugs and the risk of myocardial infarction. [12-17] 


\section{Different study designs within pharmacoepidemiology}

Different study designs can be applied within pharmacoepidemiology, all with their own specific indications, advantages and disadvantages. Within this article we provide an overview on the different study designs and if available give examples on studies conducted in children.

Although the RCT is considered to be the gold standard to study the association between drug exposure and the outcome of interest, it will not be discussed in this review as this paper only discusses study designs related to observational research.

\section{Case reports}

Case reports are reports of individual patients who experience some kind of unexpected event while taking a particular drug. Generally, no definitive inference about causality can be made, although individual reports have other uses, including alerting doctors to the possible existence of a reaction (signal generation) and helping characterize the reaction. $[8,18]$

Examples of case reports are the reports on tardive dyskinesia observed in children treated with haloperidol for Tourette syndrome. [19-21] Also the numerous case reports on serotonin syndrome, a potentially life-threatening condition, in children being treated with selective serotonin reuptake inhibitors triggered the need to study the safety of antidepressants in children. [22, 23]

\section{Case series}

Case series are collections of individual case reports who have had the same exposure or who have presented with the same outcome. Often they are collections of case reports over a fairly short period of time. Although data in case series are more numerous than in case reports, here as well, they cannot be used to test for the presence of causality as there is the lack of an appropriate comparison group. $[8,18]$ Examples of case series in children are the case series on the association between the use of dextromethorphan and serotonin syndrome. [24]

Although case series cannot investigate causality, they are important to detect potential safety signals, which need to be tested with more rigorous study designs such as case control or cohort studies.

\section{Cross sectional studies}


Cross-sectional studies measure both the exposure and disease in an individual or population at the same specific point in time. These studies can also explore the role of risk factors in the cause of a disease. Because an individual's exposure and disease status are examined at the same point in time, it is impossible to say which came first. For this reason, no conclusion about risk or cause can be made. Cross sectional studies offer a quick, easy and inexpensive method of exploring the relationship between exposures and disease. In addition, cross sectional studies can provide information on the prevalence of a disease in a population as well as other variables of interest (e.g. other concomitant diseases, use of concomitant drugs). Data used in cross-sectional studies are often collected through surveys. Surveys can be done by varies ways namely self-administered, mailed, interviews (phone or face-to face) or via the computer. There are two crucial methodological issues when conducting a survey namely the questionnaire design (open vs. closed questions) and the sampling strategy to get a representative sample of the population of interest. $[8,18]$

An example of a cross sectional study conducted in children is the study by Lin et al on the home environmental risk factors and the child's asthma status. In this study, households with children 1-17 age were requested to complete a questionnaire on home environmental risk factors and in addition were questioned about the child's asthma status. [25] Another example is the study by Liberman et al, who studied the prevalence of antihypertensive, antidiabetic and lipid lowering drugs in a population of children aged between $6-18$ years using data from a prescription claims data in the US. [26]The method used in this study was somewhat different from a classical cross sectional study as the prevalence of use was estimated on the $1^{\text {st }}$ day of each month for a total of 30 consecutive months. This is called a serial cross sectional study.

\section{Cohort study}

A cohort study is a study where a group of people with a particular disease or taking a particular drug are followed up for the occurrence of an outcome of interest. [8, 18, 27, 28] This outcome of interest will be a potential side effect in the case of safety studies. Cohort studies are generally used to compare exposed to unexposed patients, although they can also be used to compare one exposure to another. From the perspective of timing, data can be collected prospectively or retrospectively. Fig 1 and Fig 2 In prospective studies, data will be collected over time whereas in a retrospective cohort study, data have already been collected for other purposes.

As exposed and unexposed individuals are followed over time, incidence rates can be calculated within a cohort study which enables estimation of risk such as the relative and attributable risk.

Cohort studies have as big advantage that they can study rare exposures, can study multiple outcomes and that time to event analysis is possible. Disadvantages of cohort studies are that 
they are lengthy and expensive, may require very large samples, are not suitable for rare diseases or for diseases with long latency. The first two arguments do not longer hold when automated databases are as datasource, as all required information is already collected and available in the database at the time the study starts.

The principles of cohort studies can best be explained by some examples. van Staa et al conducted a retrospective cohort study using data from the GPRD (general practice research database) in the UK to study whether the use of inhaled corticosteroids (ICS) is associated with an increased risk of fractures. [29] The cohort consisted of a group of children treated with ICS and an asthma reference group of children being treated with bronchodilators excluding ICS. These patients were followed over time until the occurrence of a fracture or the end of the study. No association between the use of ICS and an increased risk of fractures could be identified. Sturkenboom et al. conducted a cohort study on the association between the use of niflumic acid and the incidence of mucocutaneous reactions in children. [30] Here as well, date from a primary care database, namely the Pedianet database in Italy was used. The cohort consisted of 3 groups of children namely children being treated with niflumic acid, other NSAIDs or acetaminophen. The children were followed until the occurrence of a mucocutaneous reaction. No association between the use of niflumic acid and the mucocutaneous reaction could be found in comparison to other NSAIDs or acetaminophen.

Although cohort studies are often used to study the association between the exposure of interest and a specific outcome, they can also be used to study drug utilization patterns over time. In addition, use of specific drugs might be used as a proxy for the prevalence of the underlying disease in children (e.g. use of anti-asthmatic drugs as proxy for prevalence of asthma). [31] Numerous studies on drug use in children have been done so far. [32-34]

As part of the TEDDY project, we investigated the prevalence of drug use in children from 2000 until 2005 using 3 primary care databases in the UK, The Netherlands and Italy. [35] Especially anti-infective, dermatological and respiratory drugs were widely prescribed for all age categories. The prevalence of drug use was the highest for the youngest age categories (age $<2$ years)

\section{$\underline{\text { 5. Case control studies }}$}

Case control studies are traditionally designed to investigate causes of rare diseases or to investigate multiple exposures. The cases are compared to healthy controls (not having the outcome) to determine the association between the possible risk factors and the disease under study. Fig 3 In pharmacoepidemiology, the cases are patients who have had a particular adverse event. The controls are drawn from those who do not have the adverse event but otherwise come from a similar population. Case control studies obtain their information on drug exposure retrospectively. Information on past exposure is obtained by abstracting medical records or by administering questionnaires or interviews. As such, case 
control studies might be subject to limitations in the validity of retrospectively collected exposure information (recall bias). [8] This bias does not hold when using prescription or pharmacy dispensing databases where information on drug prescription or drug dispensing is automatically collected in the database. Another potential limitation is the risk of selection bias where selection of cases and control might be triggered by knowledge of previous drug exposure. For this reason, validation of cases and sampling of controls should ideally be done by researchers blinded to the individual's drug exposure. As the case control studies do not provide information on the incidence rate of a disease in the exposed and unexposed individuals, relative risks cannot be calculated. Generally, the odds ratio is reported which is a close estimate of the relative risk when the disease is rare.

One of the most famous case control studies is the study by Herbst et al who studied the association between the use of diethylstilbestrol (DES) in pregnant women for the prevention of spontaneous abortion and the risk of vaginal cancer in the off-spring. [11] This study included only 8 cases for which 4 age matched controls per case were selected - this small sample size number was sufficient to reach statistical significance, as the association between the exposure and the outcome was very strong (seven of the 8 cases, but none of the 32 matched controls had been prenatally exposed to DES.

\section{$\underline{6 .}$ Nested case control study}

A specific type of a case control study is the nested case control study, whereby a case control approach is embedded within an established cohort. This approach guarantees that cases and controls represent random samples from the same study base which might be a weakness in the standard case control approach. Mikaelof et al conducted a case control study to study the association between the use of NSAIDs and the risk of skin and soft tissue complications in patients with varicella disease. [36] The cohort consisted of children diagnosed with varicella. Within this cohort, cases of skin and soft tissue complications were identified. For each case, up to 10 controls were sampled from the same cohort, matched on age and practice. The study showed a 4 -fold increased risk of skin or soft tissue complications in children treated with NSAIDs.

\section{Databases for pharmacoepidemiological research:}

\section{Spontaneous Reporting Database}

Health care providers are encouraged to report each suspicion of an adverse drug reaction (ADR) to their national monitoring system. Data from these national monitoring centers are pooled in the WHO VigiBase database. Although the spontaneous reporting system is prone to biases such as selective and underreporting and in addition lacks denominator data such 
as the total number of users, the system has been proven to be valid for safety signal generation. [37]

\section{Automated databases}

As the incidence of adverse events is often low (incidence rates $<1 / 10000$ ) and might only arise in the long term, huge sample sizes with considerate follow-up time are needed to conduct pharmacoepidemiological research. This explains why in recent years, pharmacoepidemiological research is mainly conducted using computerized health care data. The ideal database would include records from both in and outpatient care, emergency care, all laboratory and radiological tests as well as data on prescribed and over the counter medication. [8] In practice, based on the existing health policies, claims databases are used in the US and health care databases in Europe. Health care databases consist of pharmacy databases, primary care databases and hospital databases. The biggest advantage of these databases is their very large sample size and the relatively inexpensive use. The major weakness is the uncertain validity of diagnosis data which especially holds for the claims databases. [7] Neubert et al, made an inventory of all databases available for pediatric research in Europe. A total of 25 data sources from 12 European countries were identified of which the majority is suitable for paediatric pharmacoepidemiological research. [38]

Although the size of the inidiviudal automated databases is quite large, the study might still be underpowered to detect very rare side effects (e.g.1/100.000) or side effects that only occur long term after drug exposure. The power can further be increased by combining the data from the different country specific databases into one analysis set - this is nowadays one of the new developments within the field of pharmacoepidemiological research. Within one country or region, linkage of various databases might as well be appropriate, not to increase the sample size, but to collect all available data on one single individual. Primary care databases for instance might lack information on pathology data, hospital admission and hospital drug use. Linking all the available databases through a patient unique key provides the researcher with all necessary data. This principle of database linkage is common practice in the studies on the potential teratogenic effects of a drug where a prescription database is linked to a pharmacy database and a birth registry.

\section{Items to consider when conducting pharmacoepidemiology in children:}

\section{Off label and unlicensed drug use in children}

Medicines are frequently prescribed to children without being approved for the paediatric population which makes them off-label. [39] Studies have shown that the proportion of offlabel use in children might vary between 10 to $30 \%$ depending on the population being studied and the definitions that had been used. [40-42] As part of the TEDDY network, a 
consensus was reached on the definition of off label use being the following: "all use of a marketed drug not detailed in the summary of product characteristics including therapeutic indication, use in age-subsets, appropriate strength, pharmaceutical form and route of administration". [39] Research on the association between off-label status and the risk of specific side effects is scarce. [43]

\section{$\underline{\text { Age and maturation }}$}

As maturation plays a crucial role in the pharmacology of a drug, it is important to check whether the risk estimates between the drug exposure and the adverse drug reaction fluctuates by age. In paediatric drug research, it is recommended not only to study the effect of a drug in the paediatric population as a whole ( $0-18$ years), but as well to repeat the analysis within different age categories eventually the ones suggested by the International Conference on Harmonisation. [44]

\section{$\underline{\text { Gender }}$}

Sex differences in drug use have been reported in children. [32, 35]. Especially prior to puberty, the prevalence rate of drug use is higher in boys compared to girls. It is unclear whether this can be explained by a gender difference in the incidence of the disease or whether symptoms are perceived differently by parents or health care professionals. For certain diseases such as asthma, it is known that the prevalence is higher in boys. Under influence of hormones around puberty, the incidence rate of asthma changes and becomes less prominent in boys. [45] As gender might modify the association between the drug and the outcome of interest, it is recommendable to conduct additional analysis, stratifying according to gender.

\section{Pharmacovigilance during pregnancy:}

Safety disasters - such as phocomelia in children born from women who used thalidomide during pregnancy for the treatment of morning sickness and the high risk of vaginal cancer in the offspring of mothers treated with DES for the prevention of spontaneous abortion stressed the importance of the safety of a drug when used during pregnancy. [10, 11] As pregnant women are often excluded from clinical trials, the potential of teratogenesis is often uncertain when new drugs are introduced to the market. Record linkage studies are important to study the potential teratogenic effects of a drug. In this type of studies, the prescription database is linked to a pregnancy - outcome database. This type of study is described in the paper by Colvin et al, where an Australian prescription database is linked to a database with 
all pregnancies, the birth and death registration database and finally the birth defect registry. They found that women who were dispensed a drug during pregnancy were $14 \%$ more likely to have a registered birth defect. [46]

\section{Choosing the optimal pharmacoepidemiological study design}

The choice of the most appropriate study design to be used will be driven by the type of research question. Among the different study designs in pharmacoepidemiological research, cohort and case control studies have the highest level of hierarchy to establish causality, especially if all efforts are taken to reduce the risk of bias and confounding. Case reports and case series have the lowest level of hierarchy to establish causality as they do not have comparator groups.

Case records, case series as well as the analysis of spontaneous ADR reporting are used to detect safety signals. Cross-sectional studies are used to study the association between the exposure and the ADR, however, as exposure and ADRs are assessed the same point in time, they cannot be used to study causal associations. The causal association between the exposure and the outcome is tested by means of a case control or cohort study.

A case control study will be conducted in case of rare events, the need to study multiple exposures and if results need to be readily available. The risk of bias and confounding is higher compared to cohort studies although this argument no longer holds when using automated databases. Cohort studies will be used in case of low exposure and if one is interested in multiple outcomes. Cohort studies are more expensive and time-consuming especially as data need to be collected during follow-up, unless cohort studies are designed using data from automated databases.

\section{Conclusion}

There is a growing consensus on the need of safe and effective medicines in children which has led to a change in the regulation of new medicines both in the US and Europe. In addition, numerous other initiatives have been taken to promote the safety of medicines in children. Among the list of paediatric needs as established by the EMEA's Working Party, there is the need to study the long term safety effects of drugs frequently used in children. [6] These types of research questions can best be answered by observational studies, especially as the domain of pharmacoepidemiology nowadays has access to automated databases with large sample size and long term follow-up. Very few observational studies have been done so far, but this will probably change in the coming years as a result of the growing attention on the need of safe drugs in children. As observational research will become more and more important, it is crucial to further built on competence to guarantee well conducted research and the potential for further development. 


\section{References}

1. WHO, Promoting safety of drugs in children. WHO Press 2007, 2007.

2. Kearns, G.L., et al., Developmental pharmacology--drug disposition, action, and therapy in infants and children. N Engl J Med, 2003. 349(12): p. 1157-67.

3. EMEA. [cited; Available from: www.emea.europe.eu.

4. FDA. Paediatric drug development. [cited.

5. EMEA. Medicines for children - EU paediatric network. [cited; Available from: www.emea.europa.eu/htms/human/paediatrics.network.htm.

6. $\quad$ EMEA, Inventory of paediatric needs.

7. Hartzema, A.G., H.H. Tilson, and K.A. Chan, Pharmaco-epidemiology and Therapeutic Risk Management. 2008: Harvey Whitney Books Company.

8. Strom, B.L. and S.E. Kimmel, Textbook of pharmacoepidemiology, ed. L. John Wiley \& Sons. 2006.

9. Teddy. [cited; Available from: www.teddynoe.org.

10. Taussig, H.B., A study of the German outbreak of phocomelia. The thalidomide syndrome. Jama, 1962. 180: p. 1106-14.

11. Herbst, A.L., H. Ulfelder, and D.C. Poskanzer, Adenocarcinoma of the vagina. Association of maternal stilbestrol therapy with tumor appearance in young women. N Engl J Med, 1971. 284(15): p. 878-81.

12. Graham, D.J., et al., Risk of acute myocardial infarction and sudden cardiac death in patients treated with cyclo-oxygenase 2 selective and non-selective non-steroidal anti-inflammatory drugs: nested case-control study. Lancet, 2005. 365(9458): p. 475-81.

13. Hippisley-Cox, J. and C. Coupland, Risk of myocardial infarction in patients taking cyclooxygenase-2 inhibitors or conventional non-steroidal anti-inflammatory drugs: population based nested case-control analysis. Bmj, 2005. 330(7504): p. 1366.

14. Johnsen, S.P., et al., Risk of hospitalization for myocardial infarction among users of rofecoxib, celecoxib, and other NSAIDs: a population-based case-control study. Arch Intern Med, 2005. 165(9): p. 978-84.

15. Levesque, L.E., J.M. Brophy, and B. Zhang, The risk for myocardial infarction with cyclooxygenase-2 inhibitors: a population study of elderly adults. Ann Intern Med, 2005. 142(7): p. 481-9.

16. Ray, W.A., et al., COX-2 selective non-steroidal anti-inflammatory drugs and risk of serious coronary heart disease. Lancet, 2002. 360(9339): p. 1071-3.

17. Solomon, D.H., et al., Relationship between selective cyclooxygenase-2 inhibitors and acute myocardial infarction in older adults. Circulation, 2004. 109(17): p. 2068-73.

18. Torrence, M.E., Understanding Epidemiology. 1997: Mosby.

19. Caine, E.D., et al., Gilles de la Tourette's syndrome, tardive dyskinesia, and psychosis in an adolescent. Am J Psychiatry, 1978. 135(2): p. 241-3.

20. Mizrahi, E.M., D. Holtzman, and B. Tharp, Haloperidol-induced tardive dyskinesia in a child with Gilles de la Tourette's disease. Arch Neurol, 1980. 37(12): p. 780.

21. Petty, L.K. and C.J. Spar, Haloperidol-induced tardive dyskinesia in a 10-year-old girl. Am J Psychiatry, 1980. 137(6): p. 745-6.

22. Likasitwattanakul, S., Serotonin syndrome: a case report. J Med Assoc Thai, 2005. 88(7): p. 993-6.

23. Phan, H., et al., Serotonin syndrome following a single $50 \mathrm{mg}$ dose of sertraline in a child. Clin Toxicol (Phila), 2008. 46(9): p. 845-9.

24. Ziaee, V., et al., Side effects of dextromethorphan abuse, a case series. Addict Behav, 2005. 30(8): p. 1607-13.

25. Lin, S., et al., Self-reported home environmental risk factors for childhood asthma: a crosssectional study of children in Buffalo, New York. J Asthma, 2008. 45(4): p. 325-32.

26. Liberman, J.N., J.E. Berger, and M. Lewis, Prevalence of antihypertensive, antidiabetic, and dyslipidemic prescription medication use among children and adolescents. Arch Pediatr Adolesc Med, 2009. 163(4): p. 357-64. 
27. Ashby, D., Establishing causality in the assessment of safety of medicines for children. Acta Paediatr, 2008. 97(12): p. 1611-6.

28. Luo, X., et al., Role of pharmacoepidemiology in evaluating prescription drug safety in pediatrics. Curr Med Res Opin, 2007. 23(11): p. 2607-15.

29. van Staa, T.P., et al., Are inhaled corticosteroids associated with an increased risk of fracture in children? Osteoporos Int, 2004. 15(10): p. 785-91.

30. Sturkenboom, M., et al., Incidence of mucocutaneous reactions in children treated with niflumic acid, other nonsteroidal antiinflammatory drugs, or nonopioid analgesics. Pediatrics, 2005. 116(1): p. e26-33.

31. Furu, K., et al., Use of anti-asthmatic medications as a proxy for prevalence of asthma in children and adolescents in Norway: a nationwide prescription database analysis. Eur J Clin Pharmacol, 2007. 63(7): p. 693-8.

32. Fox, M.H., C.H. Foster, and J.M. Zito, Building pharmacoepidemiological capacity to monitor psychotropic drug use among children enrolled in Medicaid. Am J Med Qual, 2000. 15(4): p. 126-36.

33. Hugtenburg, J.G., E.R. Heerdink, and A.C. Egberts, Increased psychotropic drug consumption by children in the Netherlands during 1995-2001 is caused by increased use of methylphenidate by boys. Eur J Clin Pharmacol, 2004. 60(5): p. 377-9.

34. Thrane, N. and H.T. Sorensen, A one-year population-based study of drug prescriptions for Danish children. Acta Paediatr, 1999. 88(10): p. 1131-6.

35. Sturkenboom, M.C., et al., Drug use in children: cohort study in three European countries. Bmj, 2008. 337: p. a2245.

36. Mikaeloff, Y., A. Kezouh, and S. Suissa, Nonsteroidal anti-inflammatory drug use and the risk of severe skin and soft tissue complications in patients with varicella or zoster disease. $\mathrm{Br} \mathrm{J}$ Clin Pharmacol, 2008. 65(2): p. 203-9.

37. Lindquist, M. and I.R. Edwards, The WHO Programme for International Drug Monitoring, its database, and the technical support of the Uppsala Monitoring Center. J Rheumatol, 2001. 28(5): p. 1180-7.

38. Neubert, A., et al., Databases for pediatric medicine research in Europe--assessment and critical appraisal. Pharmacoepidemiol Drug Saf, 2008. 17(12): p. 1155-67.

39. Neubert, A., et al., Defining off-label and unlicensed use of medicines for children: results of a Delphi survey. Pharmacol Res, 2008. 58(5-6): p. 316-22.

40. Chalumeau, M., et al., [An example of hospital-based pharmacoepidemiology in paediatrics: tolerance to fluoroquinolones]. Arch Pediatr, 2004. 11(6): p. 500-2.

41. GW, T.J., et al., Unlicensed and off label prescription of drugs to children: population based cohort study. Bmj, 2002. 324(7349): p. 1313-4.

42. Mclntyre, J., et al., Unlicensed and off label prescribing of drugs in general practice. Arch Dis Child, 2000. 83(6): p. 498-501.

43. Evidence of harm from off label or unlicensed medicines in children. [cited; Available from: www.emea.europe.eu/pdfs/human/phvwp/23591005en.pdf.

44. EMEA. ICH Topic E 11: Clinical Investigation of Medicinal Products in the Paediatric Population. [cited; Available from: www.emea.europa.eu/pdfs/human/ich/271199en.pdf.

45. Almqvist, C., M. Worm, and B. Leynaert, Impact of gender on asthma in childhood and adolescence: a GA2LEN review. Allergy, 2008. 63(1): p. 47-57.

46. Colvin, L., et al., Pharmacovigilance in pregnancy using population-based linked datasets. Pharmacoepidemiol Drug Saf, 2009. 18(3): p. 211-25. 
Fig 1: Prospective cohort study

\section{Prospective cohort studies}
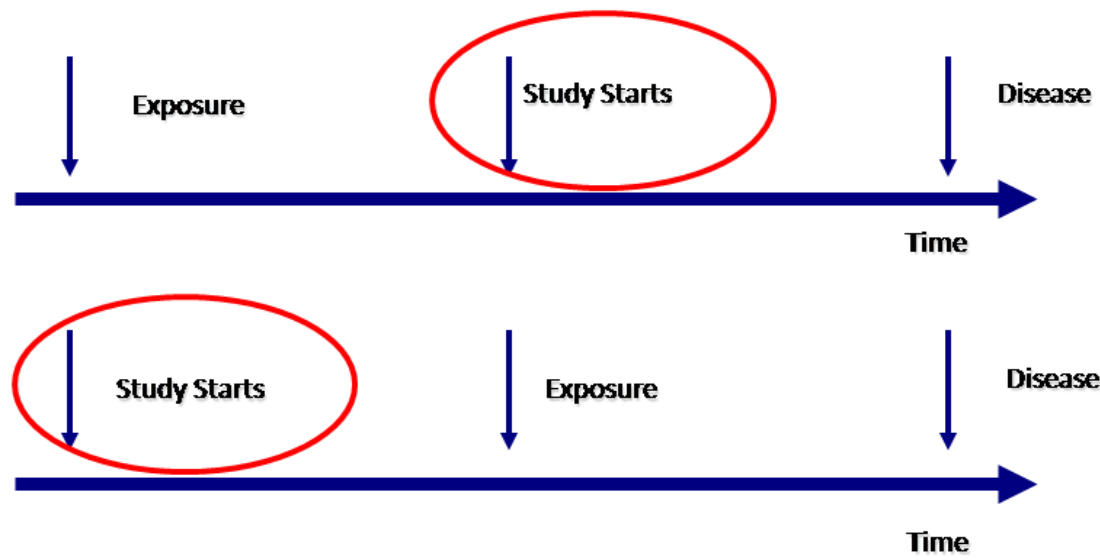

Fig 2: Retrospective cohort study

\section{Retrospective cohort studies}

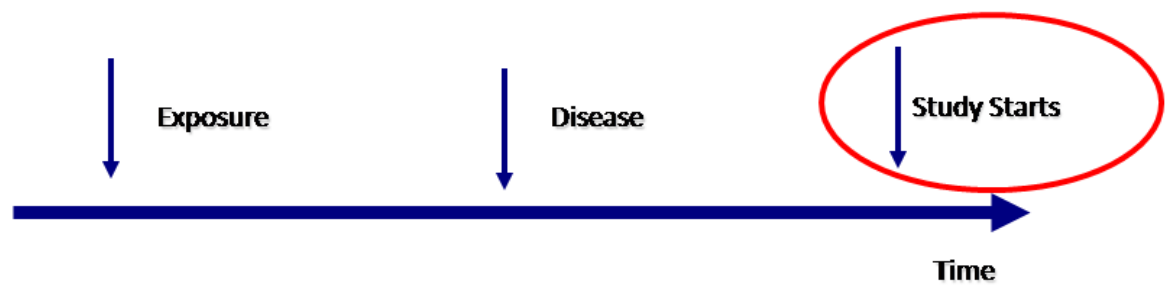




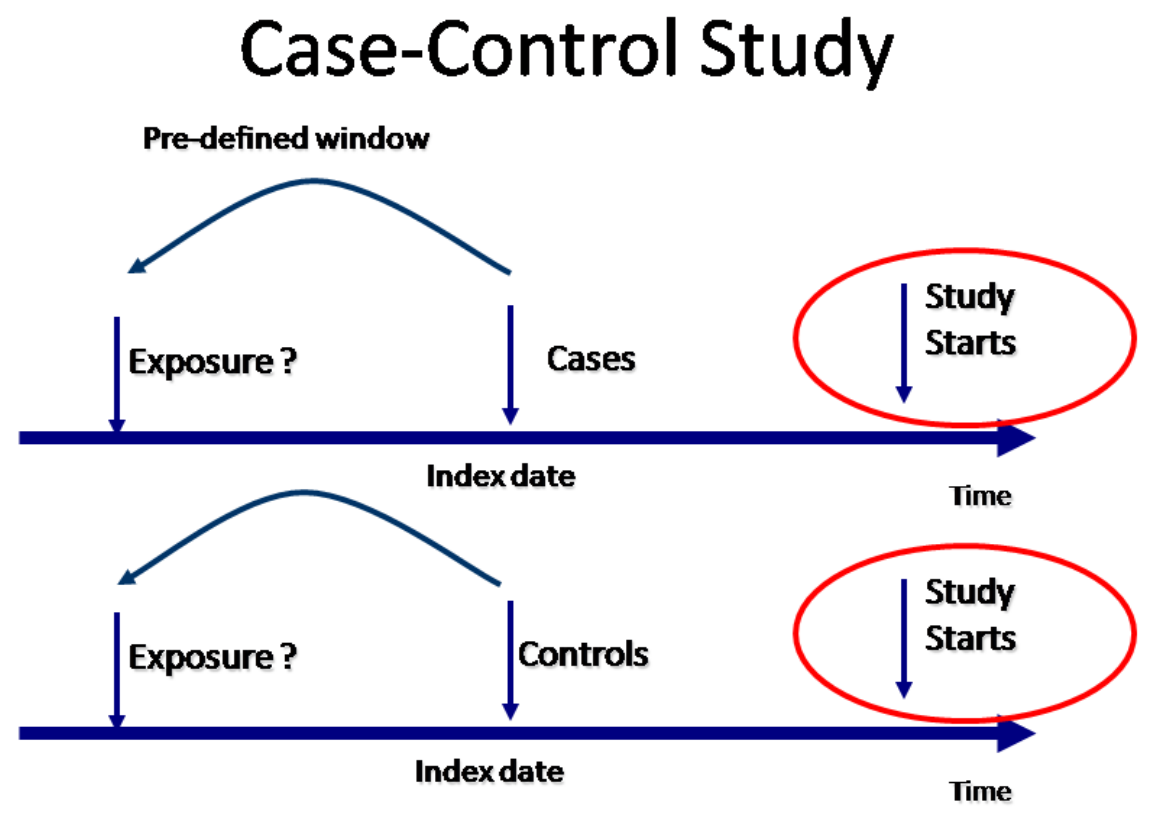

3 